\title{
Diabetes and Muscle Dysfunction in Older Adults
}

\author{
Hak Chul Jang \\ Department of Internal Medicine, Seoul National University Bundang Hospital, Seoul National University College of Medicine, Seongnam, Korea
}

\section{Corresponding Author:}

Hak Chul Jang, MD

Department of Internal Medicine,

Seoul National University Bundang

Hospital, Seoul National University

College of Medicine, 82, Gumi-ro

173beon-gil, Bundang-gu, Seongnam

13620, Korea

E-mail: janghak@snu.ac.kr

ORCID:

https://orcid.org/0000-0002-4188-6536

Received: November 3, 2019

Accepted: November 19, 2019
Aging and diabetes are both risk factors for functional decline or disability in older adults with diabetes. Recent studies demonstrated that the presence of diabetes significantly increases the risk of sarcopenia, frailty, and geriatric syndrome including falls, hospitalization, disability, and mortality in older adults. They have also suggested that sarcopenia, frailty, and geriatric syndrome should be categorized as a third category of complications in addition to the traditional microand macro-vascular complications leading to disability in older adults with diabetes. Prevention of functional decline is a crucial strategy in geriatric management. Recovery of functional independence from dependence or disability is uncommon and lengthy. Assessments of functional status and geriatric syndrome including sarcopenia or frailty should be mandatory in older adults with diabetes to promote early interventions based on physical exercise and nutrition education. This brief review discussed age-associated and diabetes-associated muscle changes and their association with functional decline.

Key Words: Skeletal muscle, Dysfunction, Diabetes mellitus, Sarcopenia, Frailty

\section{INTRODUCTION}

Diabetes, particularly type 2 diabetes (T2D), is a highly prevalent metabolic disease in older adults that is associated with microvascular and cardiovascular complications. ${ }^{1)}$ The prevalence of diabetes in people aged 65 years or older has increased to $30 \%$ in several developed countries. ${ }^{2)}$ Korea is one of the most highly ranked countries in terms of the speed of population aging. ${ }^{3)}$ In 2000, 7\% of the total Korean population was aged 65 years and increased to $14 \%$ by 2017 . The Korean Diabetes Association recently showed a steady increase in the prevalence of diabetes in the Korean population. In 2016, the prevalence of diabetes was $14.4 \%$ in Korean adults aged 30 years or older and up to $30 \%$ among those aged 65 years or over.

Several studies have shown that diabetes increased the economic, social, and health burden in older adults ${ }^{5,6)}$ and have recently drawn attention to disability and impaired quality of life in older adults with diabetes. ${ }^{7}$ The American Geriatrics Society and the American Diabetes Association published a consensus report to emphasize geriatric syndrome in diabetes care for older adults, which recommended individualized glycemic targets and diabetes management plans considering their functional status. ${ }^{8)}$ It also suggested that sarcopenia, frailty, and geriatric syndrome (including dementia and depression) be categorized as a third complication in addition to the traditional micro- and macro-vascular complications associated with functional decline. ${ }^{7,910)}$ Therefore, physical and cognitive function are of prodigious importance in the care of older people with diabetes.

A crucial strategy in geriatric management is the prevention of functional decline. Previous studies showed that the recovery of functional independence from dependence or disability was uncommon and lengthy. ${ }^{11,12)}$ However, screening of high-risk populations and timely interventions have been shown to prevent incident functional dependence or disability. ${ }^{13)}$ Increasing age and diabetes are both risk factors for functional decline and disability. Thus, understanding the mechanisms of diabetes on age-associated muscle dysfunction is crucial for early intervention and prevention of functional decline and disability in older adults with diabetes.

This brief review discussed the age-associated and diabetes-associated muscle changes and their association with functional de- 
cline. The definition and diagnosis of sarcopenia and frailty have been described in detail previously ${ }^{9,14)}$ and were not covered in this review.

\section{AGE-ASSOCIATED CHANGES IN SKELETAL MUSCLE}

Skeletal muscle has several important functions in humans, including posture, locomotion, and breathing ${ }^{15)}$ in addition to the regulation of glucose and protein metabolism and heat production. Skeletal muscle comprises $40 \%-50 \%$ of total body weight and contains $50 \%-75 \%$ of body proteins. ${ }^{16)}$ From a metabolic point of view, muscle mass depends on the balance between protein synthesis and breakdown; these processes are regulated by the multiple factors including nutrition, hormone, physical activity, exercise, and diseases (e.g., inflammation). ${ }^{16,17)}$

One of the distinctive clinical features of aging is the presence of muscle atrophy and weakness. In general, muscle mass progressively decreases after 20-30 years of age. While men have a greater muscle mass than women, the age-associated decrease in muscle mass is more rapid. ${ }^{18)}$ The reduction of muscle mass is usually associated with decreases in muscle strength and aerobic exercise capacity. The annual rate of muscle mass decline is approximately $1 \%-2 \%$, accelerates to $2 \%-3 \%$ per year after 60 years of age, and further increases after 75 years of age. ${ }^{19)}$ In contrast, fat mass, especially visceral and intermuscular fat, increases. Thus, the decrease in skeletal muscle mass and increase in ectopic fat in muscles are common elements in the age-related remodeling of body composition. Muscle is a major organ of glucose metabolism; therefore, the reduction of muscle mass may cause increased insulin resistance. ${ }^{17,20)}$ Accumulation of ectopic fat and its related adipocytokine in addition to mitochondrial dysfunction are other important factors leading to increased insulin resistance. ${ }^{21)}$

The mechanisms leading to the age-associated sarcopenia remain unclear. The proposed hypotheses include changes in muscle fiber type, apoptosis, reduced protein synthesis, hormonal changes, physical inactivity, malnutrition, and comorbidity ${ }^{16,17,20)}$ The combination of these factors may be responsible for age-associated sarcopenia. Changes in sarcopenic muscle include reduced myofiber size and number, particularly those of type II fibers and decreased numbers of type II fiber satellite cells resulting in a net change from type II to type I fibers. ${ }^{21)}$ Additionally, mitochondrial density and function in myocytes are altered. Ectopic fat infiltration (myosteatosis) and alterations of neuromuscular junction also play a crucial role in the development of sarcopenia. ${ }^{20,22)}$

Several molecular mechanisms have been suggested in sarcopenic muscle including insulin-like growth factor 1 , mammalian target of rapamycin (mTOR), myostatin/activin, and nuclear fac-
tor-kB; these complex pathways are susceptible to hormonal changes and pro-inflammatory cytokines in addition to age-associated anabolic resistance. ${ }^{20,22,23)}$

\section{DIABETES-ASSOCIATED CHANGES IN SKELETAL MUSCLE}

Bianchi and Volpato ${ }^{24)}$ recently summarized the diabetes-related changes of muscle mass and function in epidemiological studies of different populations with T2D. Cross-sectional studies showed inconsistent results in total or leg muscle mass assessed by the dual-energy X-ray absorptiometry (DXA) or computed tomography in people with diabetes (higher in two studies or lower in two studies) compared to that in non-diabetic participants. However, three prospective studies including the Health, Aging, and Body Composition study, ${ }^{25)}$ community-dwelling Chinese cohort, ${ }^{26)}$ and the Osteoporotic Fractures in Men Study ${ }^{27)}$ found an accelerated loss of muscle mass in older adults with diabetes compared to their non-diabetic counterparts. Moreover, several cross-sectional studies reported lower handgrip strength and knee extension torque in older people with diabetes despite having similar muscle mass. ${ }^{28-30)}$ Prospective cohort studies showed a steeper decline in knee extension torque. ${ }^{31,32)}$ As a consequence, the muscle quality was consistently lower in the lower extremities in most of the diabetic subjects compared to that in their non-diabetic counterparts.

In Korean older adults, the appendicular skeletal muscle mass (ASM) assessed by DXA was lower in diabetic patients than that in their non-diabetic counterparts while muscle mass index (ASM/ height ${ }^{2}$ ) was lower only in older men with diabetes. ${ }^{33)}$ Yoon et al. ${ }^{34)}$ reported that muscle mass and strength in older diabetic patients did not differ compared to those in nondiabetic subjects; however, the muscle quality was poorer and physical performance was impaired in diabetic subjects with poor glycemic control. The Korean Frailty and Aging Cohort Study (KFACS) also showed that community-dwelling older men and women with diabetes had a decreased muscle mass index (ASM/body mass index) and handgrip strength but that only women with diabetes showed decreased physical performance compared to non-diabetic participants (unpublished data). The KFACS indicated a prevalence of sarcopenia of approximately $13 \%$ in Korean older adults with diabetes according to the definition from the Asian Working Group for Sarcopenia; however, there was no significant difference in the prevalence of sarcopenia compared to that in non-diabetic participants.

The presence of diabetes accelerated the decrease of muscle mass, strength, and quality because of the associated insulin resistance and diabetes complications; ${ }^{9,35)}$ however, the underlying mechanisms of these associations remain unclear. Insulin resis- 
tance inhibits the mTOR pathway that leads to protein synthesis and decreases protein degradation. ${ }^{36)}$ Insulin resistance also increases activation of the ubiquitin-proteasome pathway, resulting in the degradation of muscle protein. ${ }^{37)}$ Chronic hyperglycemia increases the production of advanced glycation end products (AGEs). AGEs may contribute to sarcopenia through increased inflammation and endothelial dysfunction in the microcirculation of skeletal muscle; they also accumulate in the skeletal muscle and cartilage and increase stiffness in patients with diabetes. ${ }^{38,39)}$

Diabetic peripheral neuropathy is a common complication of diabetes, especially in older adults, and leads to sarcopenia in people with diabetes. Patients with peripheral neuropathy had higher calf intermuscular adipose tissue, which was associated with poor muscle strength and function. ${ }^{40)}$ Recently, Oh et al. ${ }^{41)}$ demonstrated that the muscle strength, as measured by handgrip strength, was lower in Korean men with diabetic neuropathy than that in those without neuropathy. The presence of diabetes was also associated with an increase in inflammatory cytokine levels. Increased levels are likely to be associated with both frailty and sarcopenia. ${ }^{42)}$ Moreover, systemic inflammatory cytokines, such as tumor necrosis factor and interleukin-6, have detrimental effects on muscle mass, strength, and physical performance in older adults. ${ }^{43)}$

\section{SARCOPENIA, FRAILTY, AND GERIATRIC SYNDROME IN DIABETES}

Sarcopenia is a syndrome characterized by a progressive and generalized loss of skeletal muscle mass with either muscle weakness or poor physical performance. ${ }^{14)}$ Frailty is a state of increased vulnerability to minor stressors because of decreased physiological reserve in multiple organ systems, which increases the risk of poor health outcomes including fall, hospitalization, disability, and death. ${ }^{9)}$ The KFACS demonstrated a prevalence of frailty in Korean older men and women with diabetes of $5.5 \%$ and $9.5 \%$, respectively, based on the Cardiovascular Health Study frailty phenotype criteria (unpublished data). The prevalence of frailty in women with diabetes was 2-fold higher than that in those without diabetes but the difference in the prevalence of frailty between older men with diabetes and those without diabetes was not statistically significant.

Sarcopenia and frailty have a commonality and may share similar pathways for functional decline or disability in older adults. ${ }^{9,35)}$ Sarcopenia may be an intermediate step in the development of frailty in patients with diabetes because sarcopenia is one component of the frailty phenotype. ${ }^{44)}$ Thus, sarcopenia may progress to physical frailty, then deteriorate to physical disability, and finally cause death in older adults (Fig. 1). The presence of diabetes increased the risk of sarcopenia and frailty by 2 -fold and 1.5-4-fold,

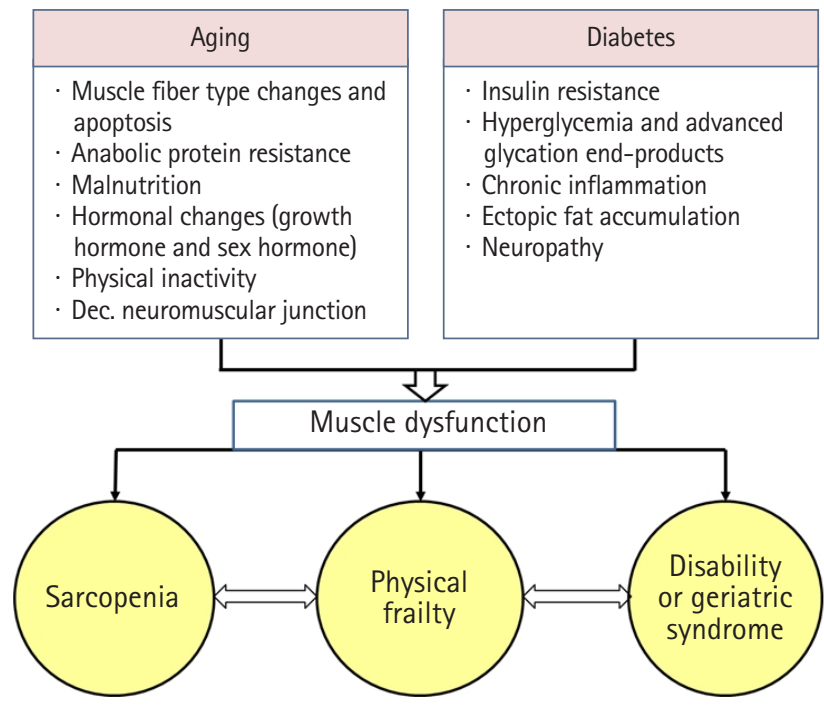

Fig. 1. Additive effects of aging and diabetes on muscle dysfunction. Sarcopenia is a major component of frailty. Diabetes accelerates the progression of sarcopenia to frailty, ultimately leading to disability, functional dependence, and death. Geriatric syndrome includes falls, incontinence, pressure ulcer, delirium, functional dependence.

respectively. ${ }^{45)}$ A meta-analysis showed that diabetes was associated with an increased odds of difficulties with activities of daily living (ADL) and instrumental activities of daily living (IADL) compared to those without diabetes (odds ratio $=1.82,95 \%$ confidence interval $1.63-2.04$ and odds ratio $=1.65,95 \%$ confidence interval $1.55-1.74$, respectively). ${ }^{7}$

Fall is a major geriatric syndrome and is detrimental to quality of life; it is associated with activity avoidance, hospitalization, and even mortality. ${ }^{46)}$ Fall is highly prevalent in older adults with diabetes, with annual incidence rates of $39 \%$ in those over 65 years of age and even higher in those with poor glycemic control and insulin treatment. ${ }^{47}$

\section{CONCLUSION}

Diabetes, sarcopenia, and frailty are associated with functional decline, disability, and mortality. Diabetes accelerates the age-associated muscle loss and progression of muscle weakness that lead to the early pathophysiologic process of frailty. Thus, the management of frailty in patients with diabetes should initially focus on sarcopenia prevention.

Assessment of functional status and screening of sarcopenia and/or frailty in older adults with diabetes should be mandatory ${ }^{8)}$ to promote early interventions based on physical exercise and nutrition education. 


\section{CONFLICT OF INTEREST DISCLOSURES}

The researcher claims no conflicts of interest.

\section{ACKNOWLEDGEMENTS}

This work was supported by a grant from the Korea Health Technology R\&D Project through the Korean Health Industry Development Institute (No. HI15-C3207).

\section{REFERENCES}

1. Whiting D, Guairquata L, Weil C, Shaw J. IDF diabetes atlas: global estimates of the prevalence of diabetes for 2011 and 2030. Diabetes Res Clin Pract 2011;94:311-21.

2. Resnick HE, Harris MI, Brock DB, Harris TB. American Diabetes Association diabetes diagnostic criteria, advancing age, and cardiovascular disease risk profiles: results from the Third National Health and Nutrition Examination Survey. Diabetes Care 2000;23:176-80.

3. Kontis V, Bennett JE, Mathers CD, Li G, Foreman K, Ezzati M. Future life expectancy in 35 industrialised countries: projections with a Bayesian model ensemble. Lancet 2017;389:1323-35.

4. Kim BY, Won JC, Lee JH, Kim HS, Park JH, Ha KH, et al. Diabetes Fact Sheets in Korea, 2018: an appraisal of current status. Diabetes Metab J 2019;43:487-94.

5. Murray CJ, Lopez AD. Measuring the global burden of disease. N Engl J Med 2013;369:448-57.

6. Sloan FA, Bethel MA, Ruiz D Jr, Shea AM, Feinglos MN. The growing burden of diabetes mellitus in the US elderly population. Arch Intern Med 2008; 168:192-9.

7. Wong E, Backholer K, Gearon E, Harding J, Freak-Poli R, Stevenson $\mathrm{C}$, et al. Diabetes and risk of physical disability in adults: a systematic review and meta-analysis. Lancet Diabetes Endocrinol 2013;1:106-14.

8. Kirkman MS, Briscoe VJ, Clark N, Florez H, Haas LB, Halter JB, et al. Diabetes in older adults. Diabetes Care 2012;35:2650-64.

9. Sinclair AJ, Abdelhafiz AH, Rodriguez-Manas L. Frailty and sarcopenia: newly emerging and high impact complications of diabetes. J Diabetes Complications 2017;31:1465-73.

10. Sinclair A, Dunning T, Rodriguez-Manas L. Diabetes in older people: new insights and remaining challenges. Lancet Diabetes Endocrinol 2015;3:275-85.

11. Boyd CM, Landefeld CS, Counsell SR, Palmer RM, Fortinsky $\mathrm{RH}$, Kresevic D, et al. Recovery of activities of daily living in older adults after hospitalization for acute medical illness. J Am Geriatr Soc 2008;56:2171-9.
12. Covinsky KE, Palmer RM, Fortinsky RH, Counsell SR, Stewart AL, Kresevic D, et al. Loss of independence in activities of daily living in older adults hospitalized with medical illnesses: in creased vulnerability with age. J Am Geriatr Soc 2003;51:451-8.

13. Pahor M, Guralnik JM, Ambrosius WT, Blair S, Bonds DE, Church TS, et al. Effect of structured physical activity on prevention of major mobility disability in older adults: the LIFE study randomized clinical trial. JAMA 2014;311:2387-96.

14. Cruz-Jentoft AJ, Bahat G, Bauer J, Boirie Y, Bruyere O, Cederholm T, et al. Sarcopenia: revised European consensus on definition and diagnosis. Age Ageing 2019;48:16-31.

15. Jang HC. How to diagnose sarcopenia in Korean older adults? Ann Geriatr Med Res 2018;22:73-9.

16. Frontera WR, Ochala J. Skeletal muscle: a brief review of structure and function. Calcif Tissue Int 2015;96:183-95.

17. Batsis JA, Villareal DT. Sarcopenic obesity in older adults: aetiology, epidemiology and treatment strategies. Nat Rev Endocrinol 2018;14:513-37.

18. Short KR, Vittone JL, Bigelow ML, Proctor DN, Nair KS. Age and aerobic exercise training effects on whole body and muscle protein metabolism. Am J Physiol Endocrinol Metab 2004; 286:E92-101.

19. Hughes VA, Frontera WR, Roubenoff R, Evans WJ, Singh MA. Longitudinal changes in body composition in older men and women: role of body weight change and physical activity. Am J Clin Nutr 2002; 76:473-81.

20. Kalyani RR, Corriere M, Ferrucci L. Age-related and disease-related muscle loss: the effect of diabetes, obesity, and other diseases. Lancet Diabetes Endocrinol 2014;2:819-29.

21. Lexell J. Human aging, muscle mass, and fiber type composition. J Gerontol A Biol Sci Med Sci 1995;50:11-6.

22. Kalinkovich A, Livshits G. Sarcopenic obesity or obese sarcopenia: a cross talk between age-associated adipose tissue and skeletal muscle inflammation as a main mechanism of the pathogenesis. Ageing Res Rev 2017;35:200-21.

23. Argiles JM, Campos N, Lopez-Pedrosa JM, Rueda R, Rodriguez-Manas L. Skeletal muscle regulates metabolism via interorgan crosstalk: roles in health and disease. J Am Med Dir Assoc 2016;17:789-96.

24. Bianchi L, Volpato S. Muscle dysfunction in type 2 diabetes: a major threat to patient's mobility and independence. Acta Diabetol 2016;53:879-89.

25. Park SW, Goodpaster BH, Lee JS, Kuller LH, Boudreau R, de Rekeneire N, et al. Excessive loss of skeletal muscle mass in older adults with type 2 diabetes. Diabetes Care 2009;32:1993-7.

26. Lee JS, Auyeung TW, Leung J, Kwok T, Leung PC, et al. The effect of diabetes mellitus on age-associated lean mass loss in 3153 
older adults. Diabet Med 2010;27:1366-71.

27. Lee CG, Boyko EJ, Barrett-Connor E, Miljkovic I, Hoffman AR, Everson-Rose SA, et al. Insulin sensitizers may attenuate lean mass loss in older men with diabetes. Diabetes Care 2011;34: 2381-6.

28. Park SW, Goodpaster BH, Strotmeyer ES, de Rekeneire N, Harris TB, Schwartz AV, et al. Decreased muscle strength and quality in older adults with type 2 diabetes: the health, aging, and body composition study. Diabetes 2006;55:1813-8.

29. Volpato S, Bianchi L, Lauretani F, Lauretani F, Bandinelli S, Guralnik JM, et al. Role of muscle mass and muscle quality in the association between diabetes and gait speed. Diabetes Care 2012;35:1672-9.

30. Kalyani RR, Tra Y, Yeh HC, Egan JM, Ferrucci L, Brancati FL. Quadriceps strength, quadriceps power, and gait speed in older U.S. adults with diabetes mellitus: results from the National Health and Nutrition Examination Survey, 1999-2002. J Am Geriatr Soc 2013;61:769-75.

31. Park SW, Goodpaster BH, Strotmeyer ES, Kuller LH, Broudeau R, Kammerer C, et al. Accelerated loss of skeletal muscle strength in older adults with type 2 diabetes: the health, aging, and body composition study. Diabetes Care 2007;30:1507-12.

32. Kalyani RR, Metter EJ, Egan J, Golden SH, Ferrucci L. Hyperglycemia predicts persistently lower muscle strength with aging. Diabetes Care 2015;38:82-90.

33. Kim KS, Park KS, Kim MJ, Kim SK, Cho YW, Park SW. Type 2 diabetes is associated with low muscle mass in older adults. Geriatr Gerontol Int 2014;14:115-21.

34. Yoon JW, Ha YC, Kim KM, Moon JH, Choi SH, Lim S, et al. Hyperglycemia is associated with impaired muscle quality in older men with diabetes: the Korean longitudinal study on health and aging. Diabetes Metab J 2016;40:140-6.

35. Jang HC. Sarcopenia, Frailty, and Diabetes in Older Adults. Diabetes Metab J 2016;40:182-9.

36. Lawrence JC Jr. mTOR-dependent control of skeletal muscle protein synthesis. Int J Sport Nutr Exerc Metab 2001;11:S17785.

37. Wang X, Hu Z, Hu J, Du J, Mitch WE. Insulin resistance accelerates muscle protein degradation: activation of the ubiquitin-proteasome pathway by defects in muscle cell signaling. Endocrinol- ogy 2006; 147:4160-8.

38. Dalal M, Ferrucci L, Sun K, Beck J, Fried LP, Semba RD. Elevated serum advanced glycation end products and poor grip strength in older community-dwelling women.J Gerontol A Biol Sci Med Sci 2009;64:132-7.

39. Payne GW. Effect of inflammation on the aging microcirculation: impact on skeletal muscle blood flow control. Microcirculation 2006; 13:343-52.

40. Tuttle LJ, Sinacore DR, Cade WT, Mueller MJ. Lower physical activity is associated with higher intermuscular adipose tissue in people with type 2 diabetes and peripheral neuropathy. Phys Ther 2011;91:923-30.

41. Oh TJ, Kang S, Lee JE, Moon JH, Choi SH, Lim S, et al. Association between deterioration in muscle strength and peripheral neuropathy in people with diabetes. J Diabetes Complications 2019;33:598-601.

42. Cesari M, Kritchevsky SB, Baumgartner RN, Atkinson HH, Penninx BW, Lenchik L, et al. Sarcopenia, obesity, and inflammation: results from the Trial of Angiotensin Converting Enzyme Inhibition and Novel Cardiovascular Risk Factors study. Am J Clin Nutr 2005;82:428-34.

43. Visser M, Pahor M, Taaffe DR, Goodpaster BH, Simonsick EM, Newman AB, et al. Relationship of interleukin-6 and tumor necrosis factor-alpha with muscle mass and muscle strength in elderly men and women: the Health ABC Study.J Gerontol A Biol Sci Med Sci 2002;57:M326-32.

44. Fried LP, Tangen CM, Walston J, Newman AB, Hirsch C, Gottdiener J, et al. Frailty in older adults: evidence for a phenotype. J Gerontol A Biol Sci Med Sci 2001;56:M146-56.

45. Bouillon K, Kivimaki M, Hamer M, Shipley MJ, Akbaraly TN, Tabak A, et al. Diabetes risk factors, diabetes risk algorithms, and the prediction of future frailty: the Whitehall II prospective cohort study.J Am Med Dir Assoc 2013;14:851.

46. Schwartz AV, Hillier TA, Sellmeyer DE, Resnick HE, Gregg E, Ensrud KE, et al. Older women with diabetes have a higher risk of falls: a prospective study. Diabetes Care 2002;25:1749-54.

47. Yang Y, Hu X, Zhang Q, Zou R. Diabetes mellitus and risk of falls in older adults: a systematic review and meta-analysis. Age Ageing 2016;45:761-7. 